\title{
ALGUNS CONHECIMENTOS MATEMÁTICOS QUE EMERGEM DA FABRICAÇÃO DA BALHESTILHA QUE SE ENCONTRA NO DOCUMENTO CHRONOGRAPHIA, REPORTORIO DOS TEMPOS...
}

\section{SOME MATHEMATICAL KNOWLEDGE THAT EMERGES FROM THE MANUFACTURE OF THE CROSS-STAFF WHICH IS IN THE DOCUMENT CHRONOGRAPHIA, REPORTORIO DOS TEMPOS...}

\author{
Antonia Naiara de Sousa Batista ${ }^{1}$ \\ Universidade Estadual do Ceará
}

\begin{abstract}
Resumo
No intuito de aproximar ambos os campos, a educação matemática e a história da matemática, encontramos, na construção da interface, a possibilidade de articulá-los. Todavia, para iniciar essa construção, é necessário partir de um objeto de estudo, que, inicialmente, pode ser um documento ou texto original, um instrumento matemático, entre outros. Nesta pesquisa, temos como objeto de estudo o documento Chronographia, Reportorio dos Tempos... (1603), de Manoel de Figueiredo (1568 - 1622), mais especificamente, o texto que apresenta a fabricação da balhestilha ou radio astronômico. Assim, este estudo tem como objetivo apresentar alguns conhecimentos matemáticos que emergem da fabricação da balhestilha. Esta pesquisa está baseada na construção de uma interface proposta por Saito e Dias (2013), na qual demos ênfase no primeiro movimento, que, a partir de uma contextualização histórica do documento no período no qual estava inserido, iniciamos uma conversa com o texto que apresenta a fabricação da balhestilha para identificar alguns conhecimentos matemáticos que estavam presentes no documento e, concomitantemente, no período. No entanto, antes da leitura do texto, realizamos um tratamento didático em relação às expressões, palavras e nomenclaturas que seriam de difícil compreensão. A partir dessa conversa com o texto, identificamos conhecimentos matemáticos do tipo, construção de retas paralelas e perpendiculares, bissetriz, trissecção de ângulos, ponto médio, além de perceber que, por meio dos seis momentos, que estão presentes na fabricação do instrumento, encontram-se construções geométricas que utilizam as definições dos Elementos de Euclides, com régua não milimetrada e compasso. Assim, este estudo visa contribuir para as pesquisas que vêm sendo desenvolvidas no âmbito da construção de uma interface, para articulação entre história e ensino de matemática.
\end{abstract}

Palavras-chave: Chronographia, Reportorio dos Tempos...; Texto da fabricação da balhestilha; Conhecimentos matemáticos.

${ }^{1}$ naiara.batista@uece.br

Número Especial - IV Seminário Cearense de História da Matemática

Boletim Cearense de Educação e História da Matemática - Volume 07, Número 20, 301 - 311 (2020)

DOI: 10.30938/bocehm.v7i20.2856 


\begin{abstract}
In order to bring together both fields, mathematics education and the history of mathematics, discover the construction of the interface with the possibility of articulating them. However, to start this construction it is necessary to start from an object of study, which can be reproduced, an original document or text, a mathematical instrument, among others. In this research, we have as object of study or document Chronographia, Reportorio dos Tempos... (1603), by Manoel de Figueiredo (1568 - 1622), more specific, or text that presents the manufacture of cross-staff or astronomical radio. Thus, this study aims to present some mathematical knowledge that emerges from the manufacture of the cross-staff. This research is based on the construction of an interface proposed by Saito and Dias (2013), in which demonstrations focusing on the first movement, from a historical context of the document in the period in which it was inserted, initiates a conversation with the text it presents the manufacture of the cross-staff to identify some mathematical knowledge that was present in the document, and at the same time, in the period. However, before reading the text, we did a didactic treatment in relation to expressions, words and nomenclatures that make understanding difficult. From this conversation with the text, we identified mathematical knowledge of the type, construction of parallel and perpendicular lines, bisector, trisection of angles, midpoint, in addition to realizing that through the six moments that are present in the manufacture of the instrument, there are geometric constructions that use the definitions of Euclid's Elements, with non-millimeter ruler and compass. Thus, this study aims to contribute to the research that has been developed in the context of building an interface, for the articulation between history and mathematics teaching.
\end{abstract}

Keywords: Chronographia, Reportorio dos Tempos...; Text of the manufacture of the cross-staff; Mathematical knowledge.

\title{
Introdução
}

A educação matemática é uma área de estudos com diferentes objetos de pesquisas, definidos sob a visão de distintos autores, entre eles, podemos destacar Garcia (2009, p. 177), que apresenta a mesma como uma "área de pesquisa que toma como foco, a sala de aula, o trabalho docente, a formação de professores e todas as questões que dizem respeito ao ensino aprendizagem de matemática" (GARCIA, 2009, p. 177).

Assim, também, a história da matemática é um campo de investigação que dispõe de distintos recursos e estratégias que visam contribuir para a construção do conhecimento matemático por meio de aspectos históricos. Saito e Dias (2013), com vistas a articular esses dois campos de conhecimento, a história da matemática e a educação matemática, propõem a construção de uma interface que promova um diálogo entre o passado e o presente para a construção do conhecimento matemático em sala de aula. 
Todavia, para a construção dessa interface, é necessário partir de um objeto de estudo, podendo ser um documento original. Neste estudo, faremos uso do documento Chronographia, Reportorio dos Tempos..., publicado em 1603, por Manoel de Figueiredo (1568 - 1622) e do instrumento denominado por balhestilha, que se encontra dentro do mesmo.

Nesse sentido, iremos optar por um estudo histórico do documento e do instrumento, pautado em uma perspectiva historiográfica atualizada, que, conforme Saito (2016, p. 260), "[...] repousa no fato de que, ao situar a matemática do passado no passado, buscando-se analisar cada etapa do desenvolvimento do conhecimento matemático segundo uma rede de relações devidamente contextualizada", podendo, emergir, questões de ordem matemática que possam ser utilizadas pelo educador matemático.

Dessa forma, este estudo é um pequeno recorte da dissertação intitulada: "Um estudo sobre os conhecimentos matemáticos incorporados e mobilizados na construção e no uso da balhestilha, inserida no documento Chronographia, Reportorio dos Tempos..., aplicado na formação de professores". Assim, temos como intuito apresentar alguns conhecimentos matemáticos que emergem da fabricação da balhestilha.

\section{O caminho metodológico}

Esta pesquisa está baseada na construção de uma interface proposta por Saito e Dias (2013), em que dois movimentos são essesciais para o processo, que são: o contexto no qual os conceitos matemáticos foram desenvolvidos; o movimento do pensamento na formação do conceito matemático. Nas pesquisas de Saito e Dias (2013), Pereira e Saito (2018) e Batista (2018), esses movimentos poderão ser estudados de maneira mais detalhada.

Portanto, este estudo está voltado para o primeiro movimento da interface, que, a partir da contextualização histórica do documento, no período no qual estava inserido, iniciamos uma conversa com o texto que apresenta a fabricação da balhestilha para identificar alguns conhecimentos matemáticos que estavam presentes no documento e, concomitantemente, no período.

O documento é do início do século XVII, escrito no português de Portugal, cujas algumas palavras possuem ortografias diferentes, sentidos distintos, principalmente, em relação à matemática. Para melhor entendermos o texto, foi realizado um tratamento 
didático em relação às expressões, palavras e nomenclaturas que seriam de difícil compreensão.

\section{Documentos e instrumentos matemáticos na construção da interface}

$\mathrm{Na}$ construção de uma interface ${ }^{2}$, ocorre a realização de dois movimentos propostos por Saito e Dias (2013), como foi citado no tópico anterior. Para que esses movimentos sejam realizados, devemos partir de um objeto de estudo, que, no caso desta pesquisa, é o documento original.

Segundo Silva (2018), esses documentos originais são dominados por essa nomenclatura porque são vistos a partir de uma perspectiva historiográfica atualizada e têm como objetivo a articulação da história com o ensino de matemática para a construção do conhecimento matemático. Na concepção de Saito (2015, p. 27), documentos podem ser “[...] não só livros e tratados, mas também cartas, manuscritos, minutas e outros documentos não só escritos, mas também aqueles da cultura material, tais como instrumentos, monumentos, máquinas, etc.”.

Portanto, Silva (2018, p. 40) resume que "[...] um documento não é apenas um papel com partes escritas, tudo pode ser um documento. Dentro deles, pode-se ter o que é chamado de "texto original", que é a sua parte escrita". Isso pode ser visto, também, neste estudo, no qual iremos dar ênfase ao estudo do texto que apresenta a fabricação da balhestilha e que se encontra na Sexta Parte do documento: Chronographia, Reportorio dos Tempos....

A balhestilha é um instrumento matemático, pois, conforme Saito (2015), esses instrumentos eram destinados a medir aquilo que Aristóteles definia por "quantidades", ou seja, ângulos e distâncias. Logo, também, pode ser utilizada na construção de uma interface, de acordo com a citação de Saito (2015) anteriormente.

Consoante a Saito (2019, p. 576), “[...] quando nos referimos a antigos instrumentos matemáticos, nós os denominamos "matemáticos" porque eles incorporam e mobilizam, entre outras coisas, conhecimentos matemáticos". Por isso, a utilização deles, na construção de uma interface, permite realizar um diálogo entre o conhecimento

\footnotetext{
${ }^{2}$ Segundo Saito e Dias (2013, p. 92), a construção da interface é uma "[...] constituição de um conjunto de ações e produções que promova a reflexão sobre o processo histórico da construção do conhecimento matemático para elaborar atividades didáticas que busquem articular história e ensino de matemática”.
} 
Alguns conhecimentos matemáticos que emergem da fabricação da balhestilha que se encontra no documento Chronographia, Reportorio dos Tempos...

Antonia Naiara de Sousa Batista

matemático que está incorporado neles e que se encontra no período no qual estavam sendo usados e a matemática presente nos livros didáticos.

\section{O documento Chronographia, Reportorio dos Tempos... e a balhestilha}

O documento Chronographia, Reportorio dos Tempos... (Figura 1) foi publicado no final do século XVI para o início do século XVII, mais especificamente, em 1603, na cidade de Lisboa, capital de Portugal. Foi escrito por Manoel de Figueiredo (1568 - 1622), mestre de matemáticas, cosmografia e navegação, tendo, portanto, o domínio não só da matemática, mas da física, da química, da hidrostática, entre outras, que subsidiavam essas classes mais específicas.

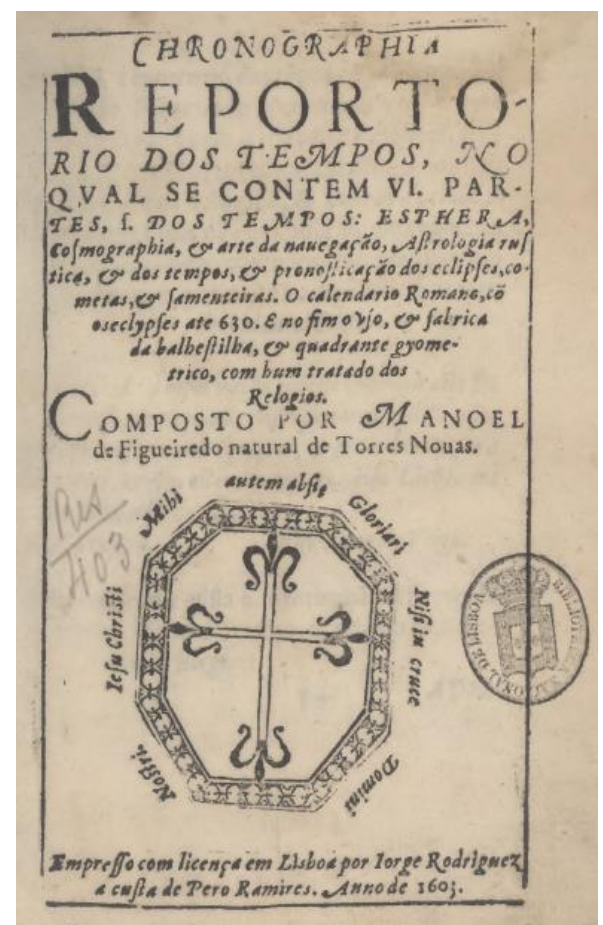

Figura 1 - Frontispício da Chronographia, Reportorio dos Tempos... (1603). Fonte: Figueiredo (1603).

A Chronographia, Reportorio dos Tempos... é um documento que congrega diversos campos do saber, que estavam em pleno desenvolvimento entre os séculos XVI e XVII, dentre eles, a astronomia, a geografia, a astrologia, a cosmografia, entre outros. Esses campos de conhecimento eram essenciais para o desenvolvimento e o progresso da navegação astronômica durante os séculos XVI e XVII.

O documento apresenta algumas particularidades, que diferem de outras Chronographias disponíveis no mesmo período, tendo a mais a presença de uma Sexta 
Parte ou Livro Sexto, no qual são apresentados a fabricação e o uso da balhestilha ou radio astronômico.

Figueiredo (1603) denomina a balhestilha por duas nomenclaturas, que estão relacionadas às finalidades do instrumento. No texto, o autor deixa claro que os astrônomos a chamaram de radio astronômico, visto que era usada para encontrar a distância entre duas estrelas (Figura 2), enquanto que balhestilha, porque o instrumento era conhecido por navegantes e tinha como objetivo conseguir a localização em alto mar, tomando a elevação do polo, pela referência da estrela Polar e a linha do horizonte.

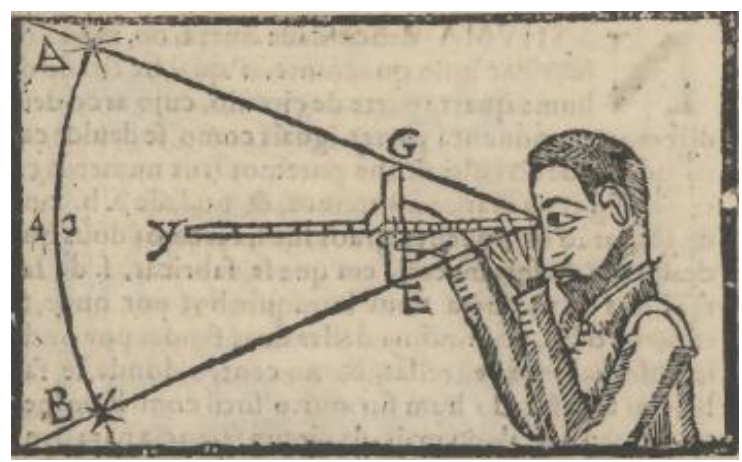

Figura 2 - Uso da balhestilha ou radio astronômico Fonte: Figueiredo (1603, f. 268)

É importante ressaltar que esse instrumento deve ser utilizado somente na vertical, como podemos ver na Figura 2. A balhestilha é um instrumento matemático simples, composto apenas por duas peças: o virote ou régua quadrada, que é uma vara comprida de faces retangulares iguais, cuja secção é um quadrado; a soalha, pinacido ou pinacidio é uma peça menor que o virote, com um orifício no centro, no qual o virote está inserido e a soalha é movimentada sobre ele (FIGUEIREDO, 1603).

\section{Conhecimentos matemáticos que podem emergir a partir de uma conversa com o texto que apresenta a fabricação da balhestilha}

O texto que apresenta a construção ou fabricação da balhestilha se encontra na Sexta Parte ou Livro Sexto do documento Chronographia, Reportorio do Tempos..., nessa parte, o instrumento, também, aparece com a denominação de radio astronômico. A fabricação descrita pelo autor está dividida em 6 momentos, como podemos ver a seguir. 
Quadro 1 - Momentos presentes na fabricação da balhestilha

\begin{tabular}{|c|l|}
\hline MOMENTOS & \multicolumn{1}{|c|}{ DESCRIÇÃO DAS ATIVIDADES } \\
\hline 1 & $\begin{array}{l}\text { Construir um quarto de círculo; Dividi-lo em 90 partes iguais; Escolha do } \\
\text { material onde será confecciondo esses procedimentos. }\end{array}$ \\
\hline 2 & $\begin{array}{l}\text { Escolha do tamanho da soalha, pinacido ou pinacidio. } \\
\text { Marcar as 90 partes em que foi dividida a circunferência em um segmento de } \\
\text { reta. }\end{array}$ \\
\hline 3 & Preparação do virote ou régua quadrada. \\
\hline 5 & Transpor as 90 divisões marcadas no segmento de reta para o virote. \\
\hline 6 & Observações sobre a confecção da solha. \\
\hline
\end{tabular}

Fonte: Figueiredo (1603)

De acordo com o Quadro 1, temos uma visão ampla dos processos abordados durante a fabricação da balhestilha. Figueiredo (1603), no início da construção, apresenta duas maneiras de iniciar a construção, sendo pela construção de um semicírculo ou por um quarto de círculo, entretanto, o autor opta pela segunda opção, descrita da seguinte forma:

\footnotetext{
[...] construa um quarto de círculo (ABC) em uma taboa. E dividiremos o arco $\widehat{\mathrm{BC}}$ pelo meio, no ponto $\mathrm{D}$. E do ponto $\mathrm{D}$ até o ponto $\mathrm{B}$ dividiremos em quarenta e cinco partes iguais. Partiremos primeiro o espaço $\widehat{\mathrm{DB}}$ em três partes iguais. $\mathrm{E}$ depois cada uma em outras três. E assim ficará partida em nove espaços. E logo cada um destes em cinco partes. E ficará partido em 45 partes iguais. E cada parte dessas partiremos pelo meio. E serão noventa partes, para o que havemos de buscar uma taboa muito plana, e lisa de cedro, ou pereiro em que tracemos a presente demonstração E depois de traçada veremos de que tamanho quero que seja, o pinacido que é o que os marinheiros chamam de soalha (FIGUEIREDO, 1603, f. 266).
}

Conforme esse trecho, alguns conhecimentos matemáticos são mobilizados, entre eles: o conhecimento de retas paralelas e perpendiculares para poder construir o quarto de círculo; a bissetriz de um ângulo; a trissecção de um ângulo; entre outros. Na Sexta Parte do documento, é possível encontrar algumas proposições que estão presentes nos Elementos de Euclides, como definições, e que dão suporte para esse passo a passo.

Além disso, ainda, na Sexta Parte, encontram-se outras proposições, assim denominadas pelo autor, mas que são conhecidas como construções geométricas realizadas com régua não milimetrada e compasso. Dando indício de que, também, nessa parte da fabricação do instrumento, foram utilizados esses procedimentos.

Isso possibilita creditar que Figueiredo (1603) utilizou, possivelmente, ferramentas como régua e compasso para dar início à fabricação do instrumento, pois Euclides já os utilizava para pôr em ação seus postulados, com um objetivo mais didático e pedagógico, eles eram empregados por restrição prática, envolvendo as circunstâncias 
de serem simples e facilitadores no seu manuseio e poderem simplificar problemas de construção (ROQUE, 2012).

No segundo momento, Figueiredo (1603, f. 266) começa a descrever o comprimento da soalha da seguinte maneira: "e suponho ser do tamanho do segmento $\overline{\mathrm{GE}}$ cujo meio será o ponto A". Nesse trecho, podemos ver que autor define o comprimento da soalha, sendo do tamanho do segmento de reta $\overline{\mathrm{GE}}$, no lado direito da Figura 3, na vertical. No entanto, Figueiredo (1603) não deixa claro o comprimento desse segmento, mas diz que o ponto A fica no meio, ou seja, A é o ponto médio do segmento, sendo $\overline{\mathrm{GE}}$ $=2 \overline{\mathrm{AE}}$.

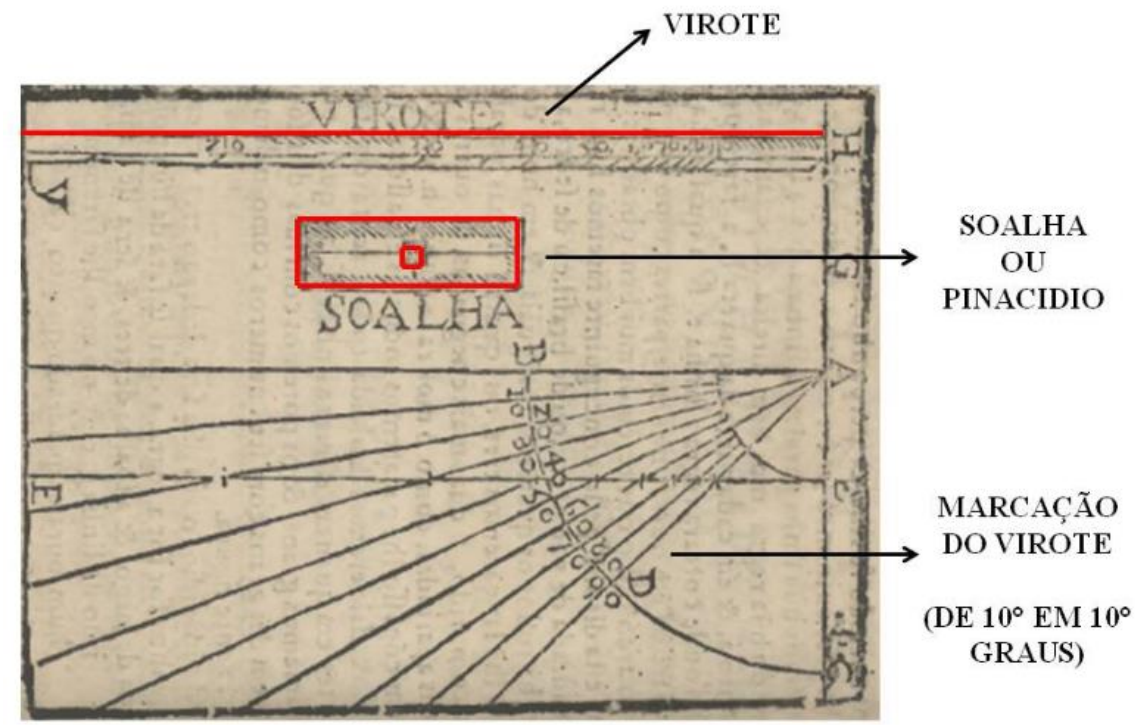

Figura 3 - Taboa da fabricação da balhestilha Fonte: Adaptado de Figueiredo (1603)

No terceiro momento, o autor sugere que, a partir da extremidade da soalha (ponto $\mathrm{E})$, seja traçado um segmento de reta $\overline{\mathrm{EF}}$, paralelo ao segmento $\overline{\mathrm{AB}}$, que corresponde a um dos lados do quarto de círculo, como podemos ver a seguir,

E do ponto E lançaremos um segmento paralelo ao segmento $\overline{\mathrm{AB}}$, o segmento $\overline{\mathrm{EF}}$. E pondo uma régua muito bem direita, e com cautela no ponto A e em cada parte do quarto $\overline{\mathrm{BA}}$ faremos divisões onde cortará a régua o segmento $\overline{\mathrm{EF}}$, no qual o segmento ficará dividido em outras noventa partes como está dividido o arco $\overline{\mathrm{BD}}$ : o que teremos muito bem operado como está dito (FIGUEIREDO, 1603, F. 267).

De acordo com o que está descrito pelo autor, iremos prolongar as retas que dividem o arco $\widehat{\mathrm{BD}}$, de maneira que elas interceptem o segmento $\overline{\mathrm{EF}}$. Veja que existe um questionamento matemático importante a se fazer nesse momento, pois o autor está 
transferindo a representação de partes do arco em segmentos lineares. É interessante ressaltar qual a relação do arco com o ângulo e com o segmento linear? No entanto, deixaremos essa discussão mais ampla para outro momento. No quarto momento, Figueiredo (1603, p. 267) relata:

E pelo conseguinte faremos uma régua quadrada de pau preto, ou de brasil, ou de cedro, a qual tenha todos os quatros lados iguais. E em um dos lados lhe lançaremos três, ou quatro segmentos paralelos ao comprido, dois mais chegados, \& outros dois mais apartados, como mostra a figura HY [segmento de reta].

Consoante a Figueiredo (1603), nesse momento, será confeccionado o virote ou régua quadrada, de pau preto, brasil ou de cedro. É importante destacar que, na visão do autor, o virote possui as quatro faces iguais, assim, chegamos à conclusão de que as faces são quadriláteros, retângulos, sendo apenas a secção do virote quadrada. No quinto momento, o autor diz:

E com um compasso lhe passaremos todos os espaços do segmento $\overline{\mathrm{EF}}$. E assim teremos dividida toda a régua quadrada em tantas partes em quantas estiver o segmento $\overline{\mathrm{EF}}$ as quais partes chamamos graus. $\mathrm{E}$ os poremos com suas divisões de dez em dez graus com seus números como demonstra a presente figura (FIGUEIREDO, 1603, F. 267).

Veja que o autor sugere a utilização do compasso para transpor as medidas do segmento $\overline{\mathrm{EF}}$ para o virote, note que essa passagem é importante, porque podemos dar continuidade ao uso do compasso, todavia, com outra finalidade. Sabemos que, no período do século XVII, existiam diferentes tipos de compassos. Por fim, no sexto momento, Figueiredo (1603, p. 267) trata da largura da soalha, como podemos ver a seguir:

\begin{abstract}
A pinacidio será de largura três vezes quanto for à régua quadrada, a qual se fará de uma polegada de largura. E a soalha de três. E será de tamanho do segmento $\overline{\mathrm{GE}}$. E no meio lhe faremos um buraco quadrado quanto caiba a régua quadrada o mais justo que puderem ser. E assim ficara feito o radio astronômico, ou balhestilha.
\end{abstract}

Diante do que autor descreveu, podemos encontrar uma relação de proporção no tocante às peças da balhestilha, pois a soalha $(\mathrm{S})$ será de largura três vezes maior quanto for a largura do virote $(\mathrm{V})$, ou seja, temos que $S_{\mathrm{L}}=3 \times \mathrm{V}_{\mathrm{L}}$, sendo $\mathrm{L}$ representando a largura. Dessa forma, este tópico apresentou algumas questões de ordem matemática que emergiram dessa conversa com o texto e que poderão ser exploradas para o encontro de algumas potencialidades didáticas. 


\section{Considerações finais}

Diante do estudo da fabricação da balhestilha, podemos perceber uma diversidade de conhecimentos matemáticos que emergiram do estudo do texto, principalmente, relacionados às construções geométricas com régua e compasso. Além disso, é interessante ressaltar que Figueiredo (1603) não construiu o instrumento diretamente físico, mas preparou uma sequência de passos destinados para construir outras balhestilhas, como se fosse um gabarito para a época.

Este estudo buscou mostrar que, a partir da construção desses instrumentos históricos, como a balhestilha, é possível encontrar conhecimentos matemáticos que são oriundos do período que estavam em uso e que, em contraste com os nossos conteúdos, que estão presentes nos livros didáticos, podem ajudar na construção do conhecimento matemático.

Assim, diante das pesquisas que vêm sendo desenvolvidas envolvendo a construção de uma interface para articulação entre história e ensino de matemática, este estudo surge no sentido de contribuir para essa articulação, trazendo outros documentos, textos e instrumentos matemáticos, que podem ser utilizados como objetos de estudo para a construção do conhecimento matemático.

\section{Referências}

BATISTA, A. N. de S. Um estudo sobre os conhecimentos matemáticos incorporados e mobilizados na construção e no uso da balhestilha, inserida no documento Chronographia, Reportorio dos Tempos..., aplicado na formação de professores. 2018. 114 f. Dissertação (Mestrado) - Curso de Pós-graduação em Ensino de Ciências e Matemática, Instituto Federal de Educação, Ciência e Tecnologia do Ceará, Fortaleza, 2018.

FIGUEIREDO, M. de. Chronographia, Reportorio dos tempos, no qual se contem VI. partes, f. dos tempos: esphera, cosmographia, e arte da navegação, astrologia rustica, e dos tempos, e pronosticação dos eclipses, cometas, e sementeiras. $\mathrm{O}$ calendario Romano, com os eclypses ate 630. E no fim o uso, a fabrica da balhestilha, e quadrante gyometrico, com hum tratado dos relogios. Lisboa. 1603.

GARCIA, Vera Clotilde Vanzetto. Fundamentação teórica para as perguntas primárias: O que é matemática? Por que ensinar? Como se ensina e como se aprende? Revista Educação, Porto Alegre, v. 32, n. 2, p.176-184, ago. 2009.

PEREIRA, Ana Carolina Costa; SAITO, Fumikazu. Os instrumentos matemáticos na interface entre história e ensino de matemática: compreendendo o cenário nacional nos 
Alguns conhecimentos matemáticos que emergem da fabricação da balhestilha que se encontra no documento Chronographia, Reportorio dos Tempos...

Antonia Naiara de Sousa Batista

últimos 10 anos. In: III Seminário Cearense de História da Matemática, 3., 2018, Fortaleza. Anais... . Fortaleza: Eduece, 2018. p. 1 - 12.

ROQUE, Tatiana. História da matemática: Uma visão crítica, desfazendo mitos e lendas. Rio de Janeiro: Zahar, 2012. 511 p.

SAITO, Fumikazu. A reconstrução de antigos instrumentos matemáticos dirigida para formação de professores. Educação: Teoria e Prática, Rio Claro, v. 29, n. 62, p.571589, 19 dez. 2019. Departamento de Educacao da Universidade Estadual Paulista UNESP. http://dx.doi.org/10.18675/1981-8106.vol29.n62.p571-589.

SAITO, Fumikazu. História e Ensino de Matemática: construindo interfaces. In: SALAZAR, Jesús Flores; GUERRA, Francisco Ugarte (Org.). Investigaciones en Educación Matemática. 1ed. Perú: Fondo Editorial PUCP, 2016, p. 253-291.

SAITO, F. Algumas considerações historiográficas. In: SAITO, Fumikazu. História da matemática e suas (re) construções contextuais. São Paulo: Editora Livraria da Física, 2015.

SAITO, F.; DIAS, M. da S. Interface entre história da matemática e ensino: Uma atividade desenvolvida com base num documento do século XVI. Ciência \&

Educação, São Paulo, v. 19, n. 1, p.89-111, 2013.

SILVA, Isabelle Coelho da. Um estudo da incorporação de textos originais para a educação matemática: buscando critérios na articulação entre história e ensino. 2018. 93 f. Dissertação (Mestrado) - Curso de Pós-graduação em Ensino de Ciências e Matemática, Instituto Federal de Educação, Ciência e Tecnologia do Ceará, Fortaleza, 2018. 